\title{
Modified FOLFIRINOX versus gemcitabine plus oxaliplatin as first-line chemotherapy for patients with locally advanced or metastatic cholangiocarcinoma: a retrospective comparative study
}

Lu Zou ${ }^{1,2,3,4+}$, Xuechuan $\mathrm{Li}^{2,3,4+}$, Xiangsong Wu ${ }^{1,3+}$, Jiujie Cui ${ }^{5+}$, Xuya Cui ${ }^{2,3,4}$, Xiaoling Song ${ }^{1,3}$, Tai Ren ${ }^{1,3}$,

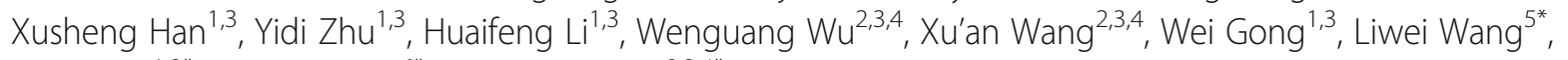
Maolan $\mathrm{Li}^{1,3^{*}}$, Wan Yee Lau ${ }^{6^{*}}$ and Yingbin Liu $2,3,4^{*}$

\begin{abstract}
Background: Gemcitabine plus platinum as the first-line chemotherapy for cholangiocarcinoma (CCA) has limited efficacy. The aim of this study was to evaluate the effectiveness of modified FOLFIRINOX (mFOLFIRINOX) compared to that of gemcitabine plus oxaliplatin (Gemox) for patients with locally advanced or metastatic CCA.

Methods: From January 2016 to December 2019, consecutive patients who were diagnosed with locally advanced or metastatic CCA were treated with either mFOLFIRINOX or Gemox as a first-line chemotherapy. The main endpoint was Progression free survival (PFS). The second endpoints were Overall survival (OS), Disease control rate (DCR) and incidence of severe toxicity (grade 3-4). Tumors were evaluated at baseline and thence every 4-6 weeks. The study was designed and carried out in accordance with the principles of the declaration of Helsinki, approved by the Ethics Committee of Xinhua Hospital Affiliated to Shanghai Jiaotong University School of Medicine (XHEC-D2020-154) and registered with ClinicalTrials.gov, number NCT04305288 (registration date: 12/03/2020).
\end{abstract}

\footnotetext{
*Correspondence: liweiwang@shsmu.edu.cn; limaolan6@163.com; josephlau@cuhk.edu.hk; laoniulyb@shsmu.edu.cn

${ }^{\dagger}$ Lu Zou, Xuechuan Li, Xiangsong Wu and Jiujie Cui contributed equally to this work.

${ }^{5}$ Department of Medical Oncology, Shanghai Cancer Institute, Renji Hospital, School of Medicine, Shanghai 200127, China

'Department of General Surgery, Xinhua Hospital Affiliated to Shanghai Jiao

Tong University School of Medicine, Shanghai 200092, China

${ }^{6}$ Faculty of Medicine, The Chinese University of Hong Kong, Shatin, Hong

Kong SAR, China

${ }^{2}$ Department of Biliary-Pancreatic Surgery, Renji Hospital, School of Medicine,

Shanghai Jiao Tong University, Shanghai 200127, China

Full list of author information is available at the end of the article
}

(C) The Author(s). 2021 Open Access This article is licensed under a Creative Commons Attribution 4.0 International License, which permits use, sharing, adaptation, distribution and reproduction in any medium or format, as long as you give appropriate credit to the original author(s) and the source, provide a link to the Creative Commons licence, and indicate if changes were made. The images or other third party material in this article are included in the article's Creative Commons licence, unless indicated otherwise in a credit line to the material. If material is not included in the article's Creative Commons licence and your intended use is not permitted by statutory regulation or exceeds the permitted use, you will need to obtain permission directly from the copyright holder. To view a copy of this licence, visit http://creativecommons.org/licenses/by/4.0/ The Creative Commons Public Domain Dedication waiver (http://creativecommons.org/publicdomain/zero/1.0/) applies to the data made available in this article, unless otherwise stated in a credit line to the data. 
Results: Of 49 patients in this study, 27 were in the FOLFIRINOX regimen group and 22 in the Gemox regimen group. There were no significant differences between groups in baseline characteristics. The DCR was $77.8 \%$ in the mFOLFIRINOX group and 63.5\% in the Gemox group. The corresponding median PFS was 9.9 months (95\% confidence interval [Cl], 7.3$12.4)$ in the mFOLFIRINOX group versus 6.4 months ( $95 \% \mathrm{Cl}, 3.6-9.2, p=0.040)$ in the Gemox group. The corresponding median OS was 15.7 months $(95 \% \mathrm{Cl}, 12.5-19.0)$ versus 12.0 months ( $95 \% \mathrm{Cl}, 9.3-14.8, p=0.099)$. Significantly more grade $3-4$ vomiting occurred in the mFOLFIRINOX than the Gemox groups (7 (25.9\%) vs $1(4.5 \%), p=0.044)$.

Conclusions: First-line mFOLFIRINOX offered more promising results in patients with advanced or metastatic CCA.

Keywords: mFOLFIRINOX chemotherapy gemcitabine cholangiocarcinoma

\section{Introduction}

Cholangiocarcinoma (CCA), which includes intrahepatic, hilar and distal CCA, is a heterogeneous group of rare tumors [1,2]. Most patients were diagnosed at advanced stage and missed the opportunity for R0 surgical resection. The prognosis for advanced CCA is less favorable with a median survival of less than 12 months and an overall survival rate (OS) of 5 years of approximately $5 \%$ [3]. Gemcitabine plus platinum, as the first-line chemotherapy for CCA, has limited efficacy (OS: 11.7 months) [4]. Gemcitabine plus oxaliplatin (Gemox) has similar efficacy compared with gemcitabine plus cisplatin (weighted median OS: oxaliplatin group vs cisplatin; 9.5 months vs 9.7 months) [5]. The progression-free survival (PFS) after second-line therapy is only about 3 months [6]. Targeted therapy and immunotherapy have the potential to become an option in the treatment of CCA [7]. However, more studies are needed to confirm the efficacy of these molecule drugs. The ESMO clinical guidelines recommend cisplatin/gemcitabine chemotherapy regimen or participation in clinical trials for patients with locally advanced or metastatic CCA [8].

Modified FOLFIRINOX (mFOLFIRINOX) regimen (irinotecan, fluorouracil, leucovorin and oxaliplatin) resulted in a longer OS than gemcitabine alone [9] (11.1 vs 6.8 months), and has become the first-line chemotherapy of metastatic pancreatic cancer. As therapeutic similarities in sensitivity to fluorouracil, platinum and gemcitabine exist between CCA and pancreatic cancer [10], mFOLFIRINOX might show better efficacy than Gemox in treating patients with CCA. Ulusakarya et al [11] had reported that the median OS of patients with advanced biliary tract cancer treated with first-line FOLFIRINOX was as long as 15 months. Recently, Angela et al [12] reported mFOLFOX (folinic acid, fluorouracil, and oxaliplatin) improved the prognosis of patients with advanced biliary tract cancer after progression on cisplatin and gemcitabine. These studies suggested that FOLFIRINOX might be a potential treatment option of CCA. This study was conducted to evaluate the effectiveness and safety of mFOLFIRINOX compared to Gemox for patients with locally advanced or metastatic CCA.

\section{Methods \\ Patients}

This is a retrospective study on consecutive patients with intrahepatic, hilar, or distal CCA at locally advanced (non-resectable) or metastatic stage who were treated with either mFOLFIRINOX or Gemox as a first-line therapy from January 2016 to December 2019 at Xinhua Hospital Affiliated to Shanghai Jiaotong University School of Medicine. All patients were diagnosed with treatment-naive CCA. Metastasis was defined as distant metastasis on medical imaging. Locally advanced disease was defined as inability to undergo radical resection in the absence of distant metastasis after assessment by an experienced surgeon. The study was censored on September 30, 2020. All patients had signed informed consent forms. The study was designed and carried out in accordance with the principles of the declaration of Helsinki, approved by the Ethics Committee of Xinhua Hospital Affiliated to Shanghai Jiaotong University School of Medicine (XHEC-D-2020-154) and registered with ClinicalTrials.gov, number NCT04305288 (registration date: $12 / 03 / 2020$ ).

\section{Treatment and assessment}

The mFOLFIRINOX regimen consisted of irinotecan $150 \mathrm{mg} / \mathrm{m}^{2}$, oxaliplatin $65 \mathrm{mg} / \mathrm{m}^{2}$, Calcium folinate 400 $\mathrm{mg} / \mathrm{m}^{2}$, fluorouracil $400 \mathrm{mg} / \mathrm{m}^{2}$ and continuous fluorouracil $2400 \mathrm{mg} / \mathrm{m}^{2}(46 \mathrm{~h})$, in a 2-week schedule. The Gemox regimen consisted of $100 \mathrm{mg} / \mathrm{m}^{2}$ oxaliplatin followed by $1000 \mathrm{mg} / \mathrm{m}^{2}$ gemcitabine on days 1 and 8 once every 3 weeks. The main endpoint was Progression free survival (PFS). The second endpoints were Overall survival (OS), Disease control rate (DCR) and incidence of severe toxicity (grade 3-4). Tumors were evaluated at baseline and thence every 4-6 weeks during treatment using magnetic resonance imaging or computed tomography. Response and progression were assessed using the Response Evaluation Criteria in Solid Tumors (RECI ST) version 1.1.

Patients underwent a complete biological examination before each treatment cycle, including full blood count 
and biochemistry of liver and kidney. Safety was evaluated using the Common Terminology Criteria for Adverse Events (version 4.0). The rates of grade 3-4 adverse events between groups were compared.

\section{Statistical analysis}

The Wilcoxon rank was used to compare continuous data with skewed distributions. Categorical variables were compared using the chi-square test or Fisher's exact test. PFS was defined as the time from diagnosis to disease progression or death, whichever occurred first. OS was defined as the time from diagnosis to death from any cause. PFS and OS were analyzed using the KaplanMeier method and the log-rank test. Univariable and multivariable analyses were performed using the Cox proportional hazards regression model to determine prognostic factors for PFS and OS. The variables that showed potential associations with OS or PFS in univariable analysis $(p<0.2)$ were further tested in multivariable analyses. All analyses were performed using SPSS version 22.0 (IBM Corp, Armonk, NY, USA). A two-sided $p$-value of $<0.05$ was considered statistically significant.

\section{Results}

\section{Patient characteristics}

From January 2016 to December 2019, 49 patients were included in the study. Table 1 summarized the baseline characteristics of these patients. Twenty-seven patients were treated with mFOLFIRINOX (15 males and $12 \mathrm{fe}$ males, median age 58 years), and 22 patients with Gemox (8 males and 14 females, median age 56.5 years). All patients had a good general condition (ECOG score of 0 or 1 ). Most patients were diagnosed with metastatic CCA (mFOLFIRINOX vs Gemox: 21 (77.8\%) in 27 vs 14 $(63.6 \%)$ in 22). Liver metastasis was common in both groups. There was no significant difference in baseline characteristics among groups.

\section{Efficacy}

The median treatment was 14 cycles for mFOLFIRINOX and 8 cycles for Gemox. Partial response (PR) occurred in $9(33.3 \%)$ patients in the mFOLFIRINOX regimen and $5(22.7 \%)$ patients in the Gemox regimen (Table 2). No patients achieved complete response (CR). The disease control rates (DCR) were 77.8 and $63.5 \%$ in the mFOLFIRINOX regimen and Gemox regimen, respectively.

Table 1 Baseline patient characteristics

\begin{tabular}{|c|c|c|c|}
\hline Characteristic & mFOLFIRINOX $(n=27)$ & Gemox $(n=22)$ & $P$ value \\
\hline \multicolumn{4}{|l|}{ Age } \\
\hline Median, range & $58(41-73)$ & $56.5(36-75)$ & 0.817 \\
\hline Sex & & & 0.181 \\
\hline Male & $15(55.6)$ & $8(36.4)$ & \\
\hline Female & $12(44.4)$ & $14(63.6)$ & \\
\hline ECOG & & & 0.407 \\
\hline 0 & $13(48.1)$ & $8(36.4)$ & \\
\hline 1 & $14(51.9)$ & $14(63.6)$ & \\
\hline Primary tumor sites & & & 0.434 \\
\hline Intrahepatic & $16(59.3)$ & $9(40.9)$ & \\
\hline Hilar & $9(33.3)$ & $11(50.0)$ & \\
\hline Distal & $2(7.4)$ & $2(9.1)$ & \\
\hline Disease status & & & 0.276 \\
\hline Locally advanced & $6(22.2)$ & $8(36.4)$ & \\
\hline Metastatic & $21(77.8)$ & $14(63.6)$ & \\
\hline \multicolumn{4}{|l|}{ Metastatic site } \\
\hline Liver & $17(63.0)$ & $13(59.1)$ & 0.782 \\
\hline Lung & $5(18.5)$ & $2(9.1)$ & - \\
\hline Bone & $1(3.7)$ & $0(0)$ & - \\
\hline Other & $1(3.7)$ & $1(4.5)$ & - \\
\hline \multicolumn{4}{|l|}{ CA19-9 } \\
\hline Median, range & $172.0(6.5-18,940)$ & $151.3(1.7-26,666)$ & 0.680 \\
\hline
\end{tabular}

ECOG Eastern Cooperative Oncology Group

Data are presented as $\mathrm{n}(\%)$ for categorical variables and as median (range) for continuous variables 
Table 2 Best tumor response according to the Response Evaluation Criteria in Solid Tumors (RECIST) 1.1

\begin{tabular}{llll}
\hline & mFOLFIRINOX $(\boldsymbol{n}=\mathbf{2 7})$ & Gemox $(\boldsymbol{n}=\mathbf{2 2})$ & $\boldsymbol{p}$ value \\
\hline Complete response & $0(0)$ & $0(0)$ & - \\
Partial response & $9(33.3)$ & $5(22.7)$ & - \\
Stable disease & $12(44.4)$ & $9(40.9)$ & - \\
Progressive disease & $6(22.2)$ & $8(36.4)$ & - \\
Objective response rate & $9(33.3)$ & $5(22.7)$ & 0.530 \\
Disease control rate & $21(77.8)$ & $14(63.5)$ & 0.276 \\
\hline
\end{tabular}

The Kaplan-Meier curves for PFS and OS were shown in Fig. 1. Median PFS was 9.9 months (95\% CI 7.3-12.4) for the mFOLFIRINOX group versus 6.4 months $(95 \%$ CI 3.6-9.2) for the Gemox group $(p=0.040)$. The corresponding median OS was 15.7 months (95\% CI $12.5-$ 19.0) versus 12.0 months ( $95 \%$ CI $9.3-14.8)$, respectively $(p=0.099)$.

Multivariable analyses are shown in Tables 3 and 4. For PFS, use of mFOLFIRINOX versus Gemox (HR = 0.353 [95\% CI, 0.180-0.694]; $p=0.003$ ), hilar CCA versus intrahepatic CCA $(\mathrm{HR}=2.149$ [95\% CI, 1.113$4.151] ; p=0.023)$, presence of liver metastasis $(\mathrm{HR}=3.096$ [95\% CI, 1.535-6.246]; $p=0.002$ ) and a high level of CA19-9 ( $\mathrm{HR}=3.622$ [95\% CI, 1.540-8.523]; $p=0.003)$ were independent prognostic factors. For OS, a high ECOG level $(\mathrm{HR}=2.148 \quad[95 \% \mathrm{CI}, 1.028-4.488] ; p=$ $0.042)$, hilar CCA versus intrahepatic CCA $(H R=2.123$ [95\% CI, 1.023-4.402]; $p=0.043$ ) and a high level of CA19-9 (HR $=4.972$ [95\% CI, 1.768-13.980]; $p=0.002)$ were independent prognostic factors.

\section{Safety}

The Grade 3-4 treatment-related adverse events were shown in Table 5 . Significantly more grade 3-4 vomiting occurred in the mFOLFIRINOX group (mFOLFIRINOX vs Gemox: $7(25.9 \%)$ vs $1(4.5 \%), p=0.044)$. However, grades 3-4 of febrile neutropenia, diarrhea and fatigue occurred only in the mFOLFIRINOX group, while grade 3-4 of thrombocytopenia occurred only in the Gemox group. Treatment was delayed because of toxicity in 11 (40.7\%) patients in the mFOLFIRINOX regimen and 3 (13.6\%) patients in the Gemox regimen.

\section{Discussion}

To our knowledge, this retrospective study was the first to directly compare the effectiveness between mFOLFIRINOX and Gemox in patients with locally advanced or metastatic CCA. These results suggested that patients received $\mathrm{mFOLFIRINOX}$ showed longer PFS than those received with Gemox as a first-line chemotherapy.

Chemotherapy is the preferred choice for locally advanced or metastatic CCA. Commonly used drugs include gemcitabine, fluorouracil and platinum [13]. The low incidence and poor prognosis of CCA resulted in few clinical trials being conducted to compare different chemotherapy regimens, and these studies often included all subgroups of biliary tract cancer. The ABC-02 [4] in 2010 established gemcitabine plus platinum as the
A

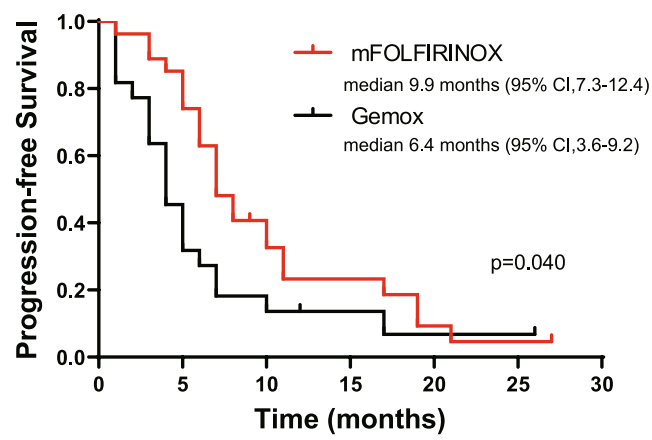

Number at risk

$\begin{array}{rrrccccc}\text { mFOLFIRINOX } & 27 & 23 & 10 & 5 & 2 & 1 & 0 \\ \text { Gemox } & 22 & 10 & 4 & 2 & 1 & 1 & 0\end{array}$

B

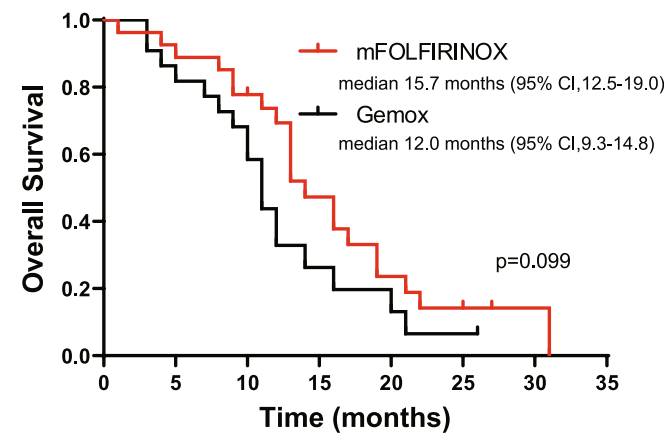

Number at risk

$\begin{array}{rllllllll}\text { mFOLFIRINOX } & 27 & 25 & 20 & 10 & 5 & 3 & 1 & 0 \\ \text { Gemox } & 22 & 19 & 14 & 4 & 3 & 1 & 0 & 0\end{array}$

Fig. 1 Progression-free survival (A) and overall survival (B) in patients receiving mFOLFIRINOX $(n=27)$ and Gemox $(n=22)$ 
Table 3 Univariable and multivariable analysis for progression-free survival (PFS)

\begin{tabular}{|c|c|c|c|c|}
\hline \multirow[t]{2}{*}{ Variables } & \multicolumn{2}{|l|}{ Univariable analysis } & \multicolumn{2}{|c|}{ Multivariable analysis } \\
\hline & HR (95\% Cl) & $P$ value & HR (95\% Cl) & $P$ value \\
\hline \multicolumn{5}{|l|}{ Treatment regimen } \\
\hline mFOLFIRINOX vs Gemox & $0.559(0.307-1.020)$ & 0.040 & $0.353(0.180-0.694)$ & 0.003 \\
\hline \multicolumn{5}{|l|}{ Age } \\
\hline$>58$ vs $\leq 58$ & $1.060(0.579-1.939)$ & 0.851 & & \\
\hline \multicolumn{5}{|l|}{ Sex } \\
\hline male vs female & $0.844(0.626-1.139)$ & 0.268 & & \\
\hline \multicolumn{5}{|l|}{ ECOG } \\
\hline 1 vs 0 & $1.220(0.664-2.242)$ & 0.523 & & \\
\hline \multicolumn{5}{|l|}{ Primary tumor sites } \\
\hline Intrahepatic & Ref & & Ref & \\
\hline Hilar & $1.599(0.869-2.944)$ & 0.131 & $2.149(1.113-4.151)$ & 0.023 \\
\hline Distal & $0.512(0.120-2.191)$ & 0.367 & $0.493(0.111-2.185)$ & 0.352 \\
\hline \multicolumn{5}{|l|}{ Disease status } \\
\hline Metastasis vs Locally advanced & $1.111(0.790-1.564)$ & 0.545 & & \\
\hline Liver metastasis & $1.658(0.891-3.088)$ & 0.111 & $3.096(1.535-6.246)$ & 0.002 \\
\hline \multicolumn{5}{|l|}{ CA19-9 } \\
\hline$>40$ vs $\leq 40$ & $1.867(0.863-4.036)$ & 0.113 & $3.622(1.540-8.523)$ & 0.003 \\
\hline
\end{tabular}

Bold values are statistically significant $(p \leq 0.05)$

Ref reference, HR hazard ratio, Cl confidence interval, ECOG Eastern Cooperative Oncology Group

Table 4 Univariable and multivariable analysis for overall survival (OS)

\begin{tabular}{|c|c|c|c|c|}
\hline \multirow[t]{2}{*}{ Variables } & \multicolumn{2}{|l|}{ Univariable analysis } & \multicolumn{2}{|c|}{ Multivariable analysis } \\
\hline & $\mathrm{HR}(95 \% \mathrm{Cl})$ & $P$ value & $\mathrm{HR}(95 \% \mathrm{Cl})$ & $P$ value \\
\hline \multicolumn{5}{|l|}{ Treatment regimen } \\
\hline mFOLFIRINOX vs Gemox & $0.595(0.312-1.135)$ & 0.099 & $0.547(0.264-1.131)$ & 0.103 \\
\hline \multicolumn{5}{|l|}{ Age } \\
\hline$>58$ vs $\leq 58$ & $1.089(0.567-2.092)$ & 0.798 & & \\
\hline \multicolumn{5}{|l|}{ Sex } \\
\hline male vs female & $0.896(0.650-1.235)$ & 0.504 & & \\
\hline \multicolumn{5}{|l|}{ ECOG } \\
\hline 1 vs 0 & $1.709(0.883-3.310)$ & 0.112 & $2.148(1.028-4.488)$ & 0.042 \\
\hline \multicolumn{5}{|l|}{ Primary tumor sites } \\
\hline Intrahepatic & Ref & & & \\
\hline Hilar & $1.609(0.827-3.129)$ & 0.161 & $2.123(1.023-4.402)$ & 0.043 \\
\hline Distal & $0.893(0.207-3.846)$ & 0.879 & $0.890(0.199-3.983)$ & 0.878 \\
\hline \multicolumn{5}{|l|}{ Disease status } \\
\hline Metastasis vs Locally advanced & $0.939(0.660-1.335)$ & 0.724 & & \\
\hline Liver metastasis & $1.393(0.716-2.710)$ & 0.329 & & \\
\hline \multicolumn{5}{|l|}{ CA19-9 } \\
\hline$>40$ vs $\leq 40$ & $3.198(1.237-8.266)$ & 0.016 & $4.972(1.768-13.980)$ & 0.002 \\
\hline
\end{tabular}

Bold values are statistically significant $(p<0.05)$

Ref reference, $H R$ hazard ratio, Cl confidence interval, ECOG Eastern Cooperative Oncology Group 
Table 5 Grade 3-4 Adverse events occurring in patients

\begin{tabular}{llll}
\hline Adverse event & mFOLFIRINOX $(\boldsymbol{n}=\mathbf{2 7})$ & Gemox $(\boldsymbol{n}=\mathbf{2 2})$ & $\mathbf{p}$ value \\
\hline Neutropenia & $\mathbf{N}(\%)$ & $8(36.4)$ & 0.407 \\
Febrile neutropenia & $13(48.1)$ & 0 & 0.107 \\
Anemia & $3(11.1)$ & $2(9.1)$ & 0.434 \\
Thrombocytopenia & $1(3.7)$ & $1(4.5)$ & 0.263 \\
Vomiting & 0 & $1(4.5)$ & $\mathbf{0 . 0 4 4}$ \\
Diarrhea & $7(25.9)$ & 0 & 0.107 \\
Peripheral neuropathy & $3(11.1)$ & $3(13.6)$ & 0.474 \\
Fatigue & $2(7.4)$ & 0 & 0.362
\end{tabular}

Bold values are statistically significant $(p \leq 0.05)$

first-line systemic therapy for biliary tract cancers. However, the median OS in that study was only 11.7 months, and up-to-now there was no standard second-line treatment. Several studies on chemotherapeutic regimens based on gemcitabine or platinum also failed to further improve survival. In a phase II trial (NCT01375972) [14], the PFS and OS of GEM/S-1 were 5.7 and 10.1 months, respectively. In another phase II study (NCT02527824) [15] using irinotecan, oxaliplatin and S1 for patients with locally advanced or metastatic biliary tract cancer, the PFS and OS were 6.8 months and 12.5 months, respectively. In another phase II clinical trial in 2018 (NCT02181634) [16], Nab-paclitaxel plus gemcitabine neither improved PFS nor OS (7.7 and 12.4 months). The effectiveness of the Gemox regimen was similar to the gemcitabine-based therapy (PFS: 6.4 months; OS: 12.0 months) in our study.

Previous studies showed that when compared with gemcitabine, FOLFIRINOX prolonged survival for patients with metastatic pancreatic cancer [9, 17]. For advanced biliary tract cancer, a retrospective study showed median OS of patients with first-line FOLFIRINOX was as long as 15 months [11]. In addition, several studies have demonstrated the efficacy and safety of FOLFIRINOX or mFOLFOX as a secondline treatment for advanced CCA [12, 18, 19]. In our study, even when mFOLFIRINOX failed to improve OS compared with Gemox (mFOLFIRINOX vs Gemox, 15.7 vs 12.0 months, $p=0.099$ ), PFS in the mFOLFIRINOX regimen was significantly prolonged compared with the Gemox regimen (mFOLFIRINOX vs Gemox, 9.9 vs 6.4 months, $p=0.040)$. Moreover, the mFOLFRINOX regimen resulted in higher ORR (33.3\% vs $22.7 \%)$ and DCR $(77.8 \%$ vs $63.5 \%)$ than the Gemox regimen. These results suggested better treatment effectiveness using mFOLFIRINOX in patients with locally advanced or metastatic CCA compared to first-line Gemox regimen.

The role of CA19-9 as a prognostic factor for CCA is controversial. In a retrospective study on 344 patients with intrahepatic CCA, CA19-9 has been found to be an independent predictive factor and it was subsequently incorporated into a prognostic score [20]. In another retrospective study on 2816 patients, elevated CA19-9 was found to be an independent risk factor for mortality in intrahepatic CCA [21]. Nevertheless, CA19-9 has also been found to be elevated in non-malignant biliary tract diseases, such as obstructive jaundice or cholangitis $[22,23]$. In this study, liver metastases and a high level of CA19-9 were determined to be independent poor prognostic factors.

mFOLFIRINOX is a four-drug regimen that has raised concerns about its adverse drug reactions. In this study, only the incidence of vomiting was increased in the mFOLFIRINOX group. More treatment delays were required in the mFOLFIRINOX group. Irinotecan and high doses of fluorouracil were the likely causes for vomiting. These results are consistent with the studies reported on pancreatic cancer $[9,17,24,25]$. In general, the toxicity of mFOLFIRINOX was tolerable.

Treatment for advanced CCA remains challengeable, and palliative therapy is the main treatment option. This study shows that mFOLFIRINOX results in promising outcomes for efficacy and safety among patients with advanced CCA. Additionally, this study could constitute the groundwork for establishing more effective sequential systemic chemotherapeutic regimen. Notwithstanding, we need to better understand which agents and which combinations of drugs are most effective and best tolerated. Targeted therapy and immunotherapy are promising therapy but needs further research.

This study has limitations. This is a retrospective study based on a variety of patient population and a small number of patients in a single hospital, which has the inherent defects of proneness to selection biases and possibilities of introducing confounding factors. Nonrandomized analysis makes the results prone to confounding and selection bias. Due to incomplete identification of adverse events, the safety profiles may have missing/ incorrect data. The patient's choice of treatment and the 
doctor's recommendation might bias the outcome. As a result of these limitations, our findings must be interpreted with caution. A phase II/III study (PRODIGE 38) [10] comparing mFOLFIRINOX with gemcitabine plus cis-platinum for locally advanced or metastatic biliary tract cancer is ongoing and may provide more convincing evidence.

In conclusion, the prognosis of patients with locally advanced or metastatic CCA was poor, first-line $\mathrm{mFOL-}$ FIRINOX offered more promising results compared to Gemox. Further prospective evaluation might provide more compelling results.

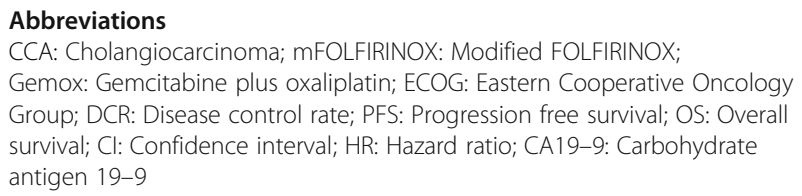

\section{Acknowledgements}

We would like to thank Dr. Xi Zhang at Xinhua Hospital Affiliated to Shanghai Jiao Tong University School of Medicine and Prof. Naiqing Zhao at Fudan university for revising this manuscript.

\section{Authors' contributions \\ Study design: YingBin Liu, Lu Zou, XiangSong Wu and Wei Gong. Data acquisition and analysis: Lu Zou, XuYa Cui, XuSheng Han, YiDi Zhu, HuaiFeng Li, JiuJie Cui, WenGuang Wu, XuAn Wang. Quality Control of Data and algorithms: XueChuan Li, XiaoLing Song, Tai Ren. Manuscript editing: Lu Zou, XuYa Cui, JiuJie Cui, MaoLan Li. Manuscript review: Wei Gong, LiWei Wang, Wan Yee Lau, YingBin Liu. The author(s) read and approved the final manuscript.}

\section{Funding}

This study was supported by the National Natural Science Foundation of China (82073206, 81773043 and 81702315), the Shuguang Program of Shanghai Education Development Foundation and Shanghai Municipal Education Commission (20SG14), the Program of Shanghai Academic Research Leader (19XD1422700), the Emerging Frontier Program of Hospital Development Center (SHDC12018107), the Clinical Science and Technology Innovation Project of Shanghai Shenkang Hospital Development Center (SHDC12019110), the Basic Research Project of Science and Technology Commission of Shanghai Municipality (No. 20JC1419100, 20JC1419101, 20JC1419102), the Shanghai Sailing Program (No.21YF1428700), and the Peak Plateau Discipline Construction Project of Shanghai Jiaotong University School of medicine (20181808).

\section{Availability of data and materials}

The data used in the current study are available from the corresponding author (laoniulyb@shsmu.edu.cn) on reasonable request.

\section{Declarations}

\section{Ethics approval and consent to participate}

The study was approved by the Ethics Committee of Xinhua Hospital Affiliated to Shanghai Jiaotong University School of Medicine (XHEC-D-2020154). All patients have been informed about the purpose and content of the study. All patients had signed informed consent forms.

\section{Consent for publication}

Not applicable.

\section{Competing interests}

All authors declare that they have no competing interests.

\section{Author details}

'Department of General Surgery, Xinhua Hospital Affiliated to Shanghai Jiao Tong University School of Medicine, Shanghai 200092, China. ${ }^{2}$ Department of Biliary-Pancreatic Surgery, Renji Hospital, School of Medicine, Shanghai Jiao Tong University, Shanghai 200127, China. ${ }^{3}$ Shanghai Key Laboratory of Biliary Tract Disease Research, Shanghai 200092, China. ${ }^{4}$ Shanghai Cancer Institute, Renji Hospital, Shanghai Jiao Tong University School of Medicine, Shanghai 200032, China. ${ }^{5}$ Department of Medical Oncology, Shanghai Cancer Institute, Renji Hospital, School of Medicine, Shanghai 200127, China. ${ }^{6}$ Faculty of Medicine, The Chinese University of Hong Kong, Shatin, Hong Kong SAR, China.

Received: 11 December 2020 Accepted: 18 June 2021 Published online: 16 July 2021

\section{References}

1. Razumilava N, Gores GJ. Classification, diagnosis, and management of cholangiocarcinoma. Clin Gastroenterol Hepatol. 2013;11(1):13-21.e1 quiz e3-4.

2. Kelley RK, Bridgewater J, Gores GJ, Zhu AX. Systemic therapies for intrahepatic cholangiocarcinoma. J Hepatol. 2020;72(2):353-63. https://doi. org/10.1016/j.jhep.2019.10.009.

3. Rizzo A, Ricci AD, Brandi G. Futibatinib, an investigational agent for the treatment of intrahepatic cholangiocarcinoma: evidence to date and future perspectives. Expert Opin Investig Drugs. 2021;30(4):317-24. https://doi. org/10.1080/13543784.2021.1837774.

4. Valle J, Wasan H, Palmer DH, Cunningham D, Anthoney A, Maraveyas A, et al. Cisplatin plus gemcitabine versus gemcitabine for biliary tract cancer. N Engl J Med. 2010;362(14):1273-81. https://doi.org/10.1056/NEJMoa 0908721.

5. Fiteni F, Nguyen T, Vernerey D, Paillard MJ, Kim S, Demarchi M, et al. Cisplatin/gemcitabine or oxaliplatin/gemcitabine in the treatment of advanced biliary tract cancer: a systematic review. Cancer Med. 2014;3(6): 1502-11. https://doi.org/10.1002/cam4.299.

6. Fornaro L, Vivaldi C, Cereda S, et al. Second-line chemotherapy in advanced biliary cancer progressed to first-line platinum-gemcitabine combination: a multicenter survey and pooled analysis with published data. J Exp Clin Cancer Res. 2015;34(1):156. https://doi.org/10.1186/s13046-015-0267-x.

7. Rizzo A, Brandi G. First-line chemotherapy in advanced biliary tract Cancer ten years after the ABC-02 trial: "and yet it moves!". Cancer Treat Res Commun. 2021;27:100335. https://doi.org/10.1016/..ctarc.2021.100335.

8. Valle JW, Borbath I, Khan SA, Huguet F, Gruenberger T, Arnold D, et al. Biliary cancer: ESMO clinical practice guidelines for diagnosis, treatment and follow-up. Ann Oncol. 2016;27(suppl 5):v28-37. https://doi.org/10.1093/a nnonc/mdw324.

9. Conroy T, Desseigne F, Ychou M, Bouché O, Guimbaud R, Bécouarn Y, et al. FOLFIRINOX versus gemcitabine for metastatic pancreatic cancer. N Engl J Med. 2011;364(19):1817-25. https://doi.org/10.1056/NEJMoa1011923.

10. Phelip JM, Edeline J, Blanc JF, Barbier E, Michel P, Bourgeois V, et al. Modified FOLFIRINOX versus CisGem first-line chemotherapy for locally advanced non resectable or metastatic biliary tract cancer (AMEBICA)PRODIGE 38: study protocol for a randomized controlled multicenter phase II/III study. Dig Liver Dis. 2019;51(2):318-20. https://doi.org/10.1016/j.dld.201 8.11.018.

11. Ulusakarya A, Karaboué A, Ciacio O, Pittau G, Haydar M, Biondani P, et al. A retrospective study of patient-tailored FOLFIRINOX as a first-line chemotherapy for patients with advanced biliary tract cancer. BMC Cancer. 2020;20(1):515. https://doi.org/10.1186/s12885-020-07004-y.

12. Lamarca A, Palmer DH, Wasan HS, Ross PJ, Ma YT, Arora A, et al. Second-line FOLFOX chemotherapy versus active symptom control for advanced biliary tract cancer (ABC-06): a phase 3, open-label, randomised, controlled trial. Lancet Oncol. 2021;22(5):690-701. https://doi.org/10.1016/S1470-2045(21 00027-9.

13. Adeva J, Sangro B, Salati M, Edeline J, la Casta A, Bittoni A, et al. Medical treatment for cholangiocarcinoma. Liver Int. 2019;39(Suppl 1):123-42. https://doi.org/10.1111/liv.14100.

14. Kang MJ, Lee JL, Kim TW, Lee SS, Ahn S, Park DH, et al. Randomized phase II trial of S-1 and cisplatin versus gemcitabine and cisplatin in patients with advanced biliary tract adenocarcinoma. Acta Oncol. 2012;51(7):860-6. https://doi.org/10.3109/0284186X.2012.682628. 
15. Yoo C, Han B, Kim HS, Kim KP, Kim D, Jeong JH, et al. Multicenter phase II study of Oxaliplatin, irinotecan, and S-1 as first-line treatment for patients with recurrent or metastatic biliary tract Cancer. Cancer Res Treat. 2018; 50(4):1324-30. https://doi.org/10.4143/crt.2017.526.

16. Sahai V, Catalano PJ, Zalupski MM, Lubner SJ, Menge MR, Nimeiri HS, et al. Nab-paclitaxel and gemcitabine as first-line treatment of advanced or metastatic cholangiocarcinoma: a phase 2 clinical trial. JAMA Oncol. 2018; 4(12):1707-12. https://doi.org/10.1001/jamaoncol.2018.3277.

17. Conroy T, Hammel P, Hebbar M, Ben Abdelghani M, Wei AC, Raoul JL, et al. FOLFIRINOX or gemcitabine as adjuvant therapy for pancreatic cancer. N Engl J Med. 2018;379(25):2395-406. https://doi.org/10.1056/NEJMoa1809775.

18. Ye LF, Ren C, Bai L, Liang JY, Hu MT, Yang H, et al. Efficacy and safety of modified FOLFIRINOX as salvage therapy for patients with refractory advanced biliary tract cancer: a retrospective study. Investig New Drugs. 2021;39(3):836-45. https://doi.org/10.1007/s10637-020-01045-7.

19. Belkouz A, De Vos-Geelen J. Mathôt RaA, et al. efficacy and safety of FOLFIRINOX as salvage treatment in advanced biliary tract cancer: an openlabel, single arm, phase 2 trial. Br J Cancer. 2020;122(5):634-9. https://doi. org/10.1038/s41416-019-0698-9.

20. Jiang W, Zeng ZC, Tang ZY, Fan J, Sun HC, Zhou J, et al. A prognostic scoring system based on clinical features of intrahepatic cholangiocarcinoma: the Fudan score. Ann Oncol. 2011;22(7):1644-52. https://doi.org/10.1093/annonc/mdq650.

21. Bergquist JR, Ivanics T, Storlie CB, Groeschl RT, Tee MC, Habermann EB, et al. Implications of CA19-9 elevation for survival, staging, and treatment sequencing in intrahepatic cholangiocarcinoma: a national cohort analysis. $J$ Surg Oncol. 2016;114(4):475-82. https://doi.org/10.1002/jso.24381.

22. Fang $\mathrm{T}$, Wang $\mathrm{H}$, Wang $\mathrm{Y}$, et al. Clinical significance of preoperative serum CEA, CA125, and CA19-9 levels in predicting the Resectability of cholangiocarcinoma. Dis Markers. 2019;2019:1-7.

23. Loosen SH, Roderburg C, Kauertz KL, et al. CEA but not CA19-9 is an independent prognostic factor in patients undergoing resection of cholangiocarcinoma. Sci Rep. 2017;7(1):1-10.

24. Kang J, Hwang I, Yoo C, Kim KP, Jeong JH, Chang HM, et al. Nab-paclitaxel plus gemcitabine versus FOLFIRINOX as the first-line chemotherapy for patients with metastatic pancreatic cancer: retrospective analysis. Investig New Drugs. 2018:36(4):732-41. https://doi.org/10.1007/s10637-018-0598-5.

25. Vivaldi C, Caparello C, Musettini G, Pasquini G, Catanese S, Fornaro L, et al. First-line treatment with FOLFOXIRI for advanced pancreatic cancer in clinical practice: Patients' outcome and analysis of prognostic factors. Int J Cancer. 2016;139(4):938-45. https://doi.org/10.1002/ijc.30125.

\section{Publisher's Note}

Springer Nature remains neutral with regard to jurisdictional claims in published maps and institutional affiliations.

Ready to submit your research? Choose BMC and benefit from:

- fast, convenient online submission

- thorough peer review by experienced researchers in your field

- rapid publication on acceptance

- support for research data, including large and complex data types

- gold Open Access which fosters wider collaboration and increased citations

- maximum visibility for your research: over $100 \mathrm{M}$ website views per year

At $\mathrm{BMC}$, research is always in progress.

Learn more biomedcentral.com/submissions 